

\title{
IMPLANTAÇÃO DE SISTEMA DE IOT PARA RASTREAMENTO DE MÁQUINAS DE TERRAPLANAGEM A SERVIÇO DE UMA INDÚSTRIA DE CELULOSE
}

\author{
IMPLANTATION OF AN IOT SYSTEM FOR TRACKING EARTHMOVING MACHINES IN \\ THE SERVICE OF A PULP INDUSTRY
}

Barbara Martins de Sousa ${ }^{1}$

Tatielle Menolli Longhini ${ }^{2}$

\author{
${ }^{1}$ Bacharel em Engenheira de Produção \\ Instituto Federal de Educação, Ciência e \\ Tecnologia de Minas Gerais - IFMG \\ Governador Valadares, MG, Brasil. \\ barbaramartins01@hotmail.com \\ 2 Meste em Administração \\ Instituto Federal de Educação, Ciência e \\ Tecnologia de Minas Gerais - IFMG \\ Governador Valadares, MG, Brasil. \\ tatielle.longhini@ifmg.edu.br
}

Recebido em: 23 abr. 2020

Aprovado em: 06 nov. 2020
Resumo: A telemetria satelital tem sido utilizada como uma alternativa para obter diferentes informações, em tempo real, de equipamentos, elevando a produtividade dos processos. Este trabalho visa implantar de sistema de loT para rastreamento em máquinas de terraplanagem a serviço de uma indústria de celulose, demonstrando os impactos de tal tecnologia. Para isso, buscou-se substituir formulário manual de controle de uso de máquinas por desenvolvimento de sistema de telemetria satelital, que permitiam o acompanhamento em tempo real dos equipamentos usados. Obteve-se êxito na implementação do sistemas e alcançou-se ganho de produtividade de 1,0\% na motoniveladora, 0,33\% na pá carregadeira e 0,81\% na retroescavadeira, comprojeção de redução de custo de $0,8 \%$ da hora-máquina - resultado replicável às outras classes de máquinas de terraplanagem. Em termos teóricos, reforçou-se a importânca do uso de sistema de loT, uma vez que possibilita controle operacional à distância, monitoramento de indicadores-chave em tempo real, levantamento de não conformidades, facilidade de armazenamento de dados e diminuição de documentos físicos e requisitou mudanças organizacionais e culturais entre os colaboradores. Trata-se de um sistema facilmente replicável a empresas com tais tipos de operação.

Palavras-chave: Telemetria satelital. Tecnologia. Produtividade. IoT.

Abstract: Satellite telemetry has been used as an alternative to obtain different information, in real time, from equipment, increasing the productivity of processes. This work aims to implement an loT system for tracking earthmoving machines in the service of a pulp industry, demonstrating the impacts of such technology. To this end, an attempt was made to substitute a manual form for controlling the use of machines by developing a satellite telemetry system, which allowed real-time monitoring of the equipment used. The systems implementation was successful and a productivity gain of $1.0 \%$ was achieved in the motor grader, $0.33 \%$ in the loader and $0.81 \%$ in the backhoe, with cost reduction projection of $0.8 \%$ of the hour. -machine - result replicable to other classes of earthmoving machines. In theoretical terms, the importance of using an IoT system was reinforced, since it allows operational control at a distance, monitoring of key indicators in real time, survey of non-conformities, ease of data storage and reduction of physical documents and requested organizational and cultural changes among employees. It is a system easily replicable to companies with such types of operations.

Keywords: Satellite telemetry. Technology. Productivity. IoT. 
1 Introdução

Métodos tradicionais de avaliação se baseiam em decisões centralizadas e subjetivas, pouco embasados por dados de desempenho. Computadores e máquinas vêm sendo incorporados para automatizar e assessorar as atividades, antes realizadas manualmente. Fato este que trouxe mudanças tanto no ponto de vista dos processos, como na maneira em que as pessoas se relacionam e se comunicam (Lemos \& Josgrilberg, 2009).

O rápido desenvolvimento de tecnologias de informação traz melhorias técnicas para análise do negócio. Com isso, a eficiência do sistema é elevada uma vez que, quando de posse de informações de qualidade, os riscos de operação são minimizados(Li et al., 2020; Ross, 2016).

Através dos dados, é possível integrar diferentes processos internos e externos à empresa devido a melhoria de comunicação e transmissão de dados (Núñez-Merino, Maqueira-Marín, MoyanoFuentes \& Martínez-Jurado, 2020; Ben-Daya, Hassini \& Bahroun, 2019; Ellis, Morris \& Santagate, 2015). Por isso, as indústrias têm investido na automação de processos, pretendendo um aumento de produtividade, considerando a rapidez e a precisão na execução das tarefas (Silveira \& Santos, 2002).

Tornou-se comum o uso de sensores e atuadores sem fio para rastreamento e monitoramento. Neste contexto, a Internet das Coisas (IOT) se destaca por proporcionar visibilidade, agilidade e adaptabilidade ao processo de gestão (Xu, He \& Li 2014). Pois permite que os gestores acessem e monitorem dados, sendo obtidas informações de qualidade para a tomada de decisão (Ben-Daya, Hassini, Bahroun, 2019; Brous \& Janssen, 2017; Hounsell et al., 2009; Ramos et al., 2008).

Ao propiciar a conectividade digital do funcionamento dos ativos, com armazenamento, análise e compartilhamento de dados, a loT facilita o planejamento, o monitoramento e a execução de processos (Ben-Daya, Hassini, Bahroun, 2019). O que permite a virtualização e a visibilidade, em tempo real, de comportamento do sistema (Verdouw, Wolfert, Beulens \& Rialland, 2016; Decker et al., 2008).

Estudos anteriores, como o de Florence e Queree (1993), já exploraram a aplicabilidade e a importância da adoção de sistemas de telemetria, por loT, para a a rastreabilidade de equipamentos e o quão necessário se faz o desenvolvimento de novas tecnologias que permitam tal monitoramento. Fato que novamente é reforçado por Ding, Jin, Li \& Feng (2020), que demarcam as dificuldades de desenvolver soluções técnicas, especialmente no uso de loT para monitoramento de equipamentos logísticos. O que requer, necessariamente, o avanço de tecnologias de loT, de informação e de comunicação, de modo que estas estejam integradas aos sistemas de gestão.

Indústrias de celulose apresentam dificuldade na implementação de sistemas de IoT, especialmente em suas atividades de terraplanagem, dadas as dificuldades técnicas de armazenamento e processamento de dados. Principalmente por tais atividades acontecerem em âmbito rural, com baixo sinal de radiofrequência para transferência de dados. 
Por isso, o propósito dessa pesquisa éimplementar um sistema de loT, para rastreamento de máquinas de terraplanagem a serviço de uma indústria de celulose, demonstrando os reflexos dessa tecnologia à produtividade do processo. Dessa forma, esse estudo será guiado pela seguinte pergunta de pesquisa: Como a implantação do sistema loT para rastreio, por telemetria satelital, pode impactar a operação de máquinas de terraplanagem a serviço de uma indústria de celulose?.

Para responder a essa questão, foi desenvolvido um estudo quali-quantitativo (Bogdan \& Biklen, 2013; Marconi \& Lakatos, 2017) em uma empresa produtora de celulose, cujo serviço de terraplanagem é realizado por uma empresa prestadora de serviço. O controle das horas trabalhadas pelas máquinas de terraplanagem era feito por um documento denominado "Parte Diária", preenchido manualmente pelo operador do equipamento. Com a proposta, visa-se desenvolver sistema de loT para rastreio das máquinas de terraplanagem e, com isso, controlar e planejar melhor o uso das máquinas e menor custo de operação, além de conseguir acessar informações em tempo real sobre diversas atividades como: localização, velocidade, disponibilidade mecânica, tempo de deslocamento, frenagens, entre outros.

Quando à organização deste artigo, após esta introdução, a segunda seção apresenta a revisão de literatura, sendo apontados os impactos do uso de ferramentas de loT no processo de gestão, bem como características do setor florestal brasileiros e as atividades de silvicultura, colheita e infraestrutura florestal, e do setor de celulose.A terceira seção descreve os passos metodológicos adotados para o estudo. A quarta seção apresenta o desenvolvimento do sistema de loT para rastreamento das máquinas de terraplanagem a serviço de uma indústria de celulose e os impactos de sua implementação. A quinta seção traz discussões entre os resultados e a teoria. Finalmente, serão apresentadas as principais conclusões e sugestões de estudos futuros na última seção.

\section{Revisão da literatura}

Esta seção contempla a abordagem de conceitos essenciais, tais como o uso de sistemas de loT e a melhorias no desempenho de gestão e características nacionais do setor florestal e de celulose.

\subsection{A adoção da Internet das Coisas (IoT) e os reflexos no desempenho de gestão}

A rastreabilidade da cadeia de suprimentos passou a ser foco do ciclo logístico, principalmente com o uso de tecnologias como: dados móveis, identificação automática de veículos e sistema de posicionamento global (GPS) (Florence, Queree, 1993). Para isso, é comum usar sistemas de radiotelemetria, que interagem com os de controle de processo e de supervisão (Greeves, 1994).

O uso de sistemas de telemetria se mostra especialmente útil na diminuição dos custos de operação, bem como no desenvolvimento de indicadores de desempenho que favorecem o monitoramento dos processos (Neto, Costa, Sousa, Amorim \& Godinho Filho, 2019; Teixeira, Oliveira \& 
Helleno, 2014). Dada sua eficiência, as organizações buscam implementá-las visando ganho de produtividade e obter melhores resultados, aproveitando bem a matéria-prima, a capacidade das máquinas, as habilidades das pessoas e o tempo, com total controle dos processos (Comunello, 2014). Com a transmissão de informações criticas, em tempo real, para o responsável da frota, é possível identificar comportamentos indevidos ou falhas no sistema (Teixeira, Oliveira \& Helleno, 2014).

No início dos anos 2000, já existiam muitos softwares de gestão de ativos, com uso de loT. Desde então, diferentes recursos foram desenvolvidos para a interpretação de dados estruturados e não estruturados. Internet das coisas se refere a objetos físicos com capacidade de transmitir dados pela internet, sendo atualmente apoiada por redes de dispositivos móveis, GPS e computação em nuvem Atzori, lera \& Morabito, 2010; Hassanain et al., 2003; Vanier, 2001).

Com a adoção do loT, o negócio pode se beneficiar de: melhores planejamentos para o uso de recursos (incorrendo em redução de custos e de tempo de execução), automação de processos, rapidez no processamento de informações, maior precisão das inspeções e das análises e redução da necessidade de inspeções físicas à vista (Rathore et al., 2016, Ahlborn et al., 2010, Ahlborn et al., 2010).

No geral, a automatização dos processos evidencia tanto a necessidade de mudança de estruturas e culturas organizacionais, quanto a de adaptação ao exercício de novas funções (Brous, Janssen \& Herder, 2019; Brous \& Janssen, 2016; Boos et al., 2013). Neste contexto, os stakeholders desejam ter acesso às informações, de modo que as decisões sejam fundamentadas pelos dados.

Especialmente aquelas relacionadas à gestão de desempenho e de manutenção de ativos e à percepção quanto à melhoria e adoção de novas funções, sendo mantido o foco no nível de serviço oferecido ao usuário (Parida et al., 2015; Ardalan et al., 1992; Koo et al., 2015; Eisenhardt \& Zbaracki, 1992). Por isso, é necessário intensificar a adoção e o estudo de loT na prática logística, de maneira a se integrar tecnologias de informação e comunicação avançadas com sistemas de gestão (Ding, Jin, Li \& Feng, 2020).

\subsection{Setor florestal e a indústria de celulose nacional}

O Brasil possui a segunda maior cobertura florestal total e a maior extensão de florestas tropicais do planeta, situando-se entre os 10 maiores países em florestas plantadas, representando 1,3\% de toda a riqueza nacional e 6,9\% do PIB industrial em 2018 (SFB, 2012; Relatório do Ibá, 2019). 0 setor ainda foi responsável pela produção de $\mathrm{R} \$ 12,80$ bilhões em tributos, US\$12,5 bilhões em receita de exportações e de 3,8 milhões de empregos diretos e indiretos, contando com uma área de 7,84 milhões de hectares de reflorestamento e é responsável por mais de 90\% de toda a madeira produzida para fins industriais (Ibá, 2018). 
Neste contexto, a indústria de celulose e papel é um dos ramos industriais que mais se destaca no mundo (Furley, Mello \& Joselaine, 2018; Rocha, 2016), sendo 36,0\% de toda a madeira produzida no Brasil utilizado para a produção de celulose e papel (Ibá, 2019). Para obter a polpa ou pasta celulósica, é necessário separar a celulose dos outros componentes vegetais (Navarro, Navarro \& Tambourgi, 2007). O processo químico de polpação kraft é a técnica mais utilizada para a obtenção da polpa celulósica (Navarro, Navarro \& Tambourgi, 2007).

Em 2017 a produção brasileira de celulose alcançou um volume de 19,5 milhões de toneladas. No entanto, no ano de 2018, foram produzidos 21,1 milhões de toneladas de celulose, uma variação de 8,0\% em relação ao ano anterior, sendo $70 \%$ destinado à exportação e 30\% destinado ao mercado doméstico. O Brasil ocupa a segunda posição de maior produtor de celulose do mundo, atrás apenas dos Estados Unidos (Ibá, 2019).

Atualmente, as maiores empresas do setor de celulose têm usado as mais modernas tecnologias para aumento de produtividade, podendo ser redefinidos sistemas e máquinas usadas (Penna, Machado \& Souza, 2011; Lopes, 2012). Por isso, busca-se a minimização dos custos de produção e da dependência da mão de obra, o que reflete em aumento de produtividade (Oliveira, Machado, Souza \& Leite, 2006; Santos, Souza, Marzano \& Minette, 2013).

\subsubsection{Silvicultura, colheita e infraestrutura florestal}

A silvicultura estuda as maneiras naturais ou artificiais de melhorar o povoamento florestal e visa manutenção, aproveitamento e uso consciente das florestas (Binkowski, 2009). O suprimento de madeira da indústria nacional de celulose provem $100 \%$ das árvores plantadas, sendo o eucalipto e o pinus os principais gêneros cultivados pela silvicultura brasileira (Fischer \& Zylbersztajn, 2012). Dentre as atividades que formam a silvicultura estão: preparo de solo, plantio, adubação e os chamados tratos culturais, que são as operações que buscam ajudar e manter o povoamento florestal (Moura, 2013).

Já a colheita florestal pode ser definida como derrubada e processamento de árvores para atender uma demanda. É um conjunto de operações de preparação e extração da madeira até o local de transporte, de acordo com a aplicação de técnicas e padrões estabelecidos, buscando o beneficiamento do produto final (Sanches, 2014). O trabalho vai desde a preparação das árvores, para o abate, até o transporte para o local de uso. Conforme a situação, o processo de colheita pode envolver também o planejamento da operação, medição, recebimento no pátio da indústria e comercialização desta madeira (Freitas, 2005).

A colheita é composta por duas etapas básicas: o corte e a extração. Conforme o grau de mecanização das máquinas e equipamentos do processo, realiza-se o corte totalmente no interior do talhão. Na etapa de extração, a madeira é removida para a margem do talhão ou pátio intermediário 
(Machado et al., 2008). Talhão é a unidade mínima de cultivo de uma propriedade desenvolvido com base em relevo e planejamento de mecanização (Dulft, 2014).

O transporte da madeira e a colheita florestal representam mais de $50 \%$ dos custos, tornandose uma das etapas mais importantes do processo. Trata-se também da etapa que determina a maior rentabilidade e a otimização das atividades reflete na melhoria da produtividade e competitividade, indispensável à sustentabilidade do negócio florestal em um mercado consumidor globalizado (Bramucci, 2001; Machado, Silva \& Pereira, 2008; Schettino, Minette \& Souza, 2015).

Para a realização do manejo florestal, é necessária a abertura da infraestrutura florestal permanente, constituída de pátios de estocagem, estradas primárias e secundárias, pontes, bueiros, sarjetas, entre outros. A infraestrutura florestal é o componente imprescindível no padrão espacial do corte seletivo de madeira, composta por estradas primárias para o acesso à área de manejo, e estradas secundárias ligando os pátios de estocagem (Pinagé \& Matricardi, 2015).

As estradas florestais são consideradas um tipo de investimento que deve englobar aspectos sociais, viabilidade técnica, menor custo de implantação e de manutenção e redução de danos ambientais (Malinovski et al., 2004). Segundo Campos (2014), a base da atividade madeireira no Brasil são as estradas de uso florestal, pois através delas é realizado o tráfego de mão de obra e dos meios de produção utilizados para a implantação, proteção, colheita e transporte da madeira.

A construção e manutenção de estradas sem planejamento pode impactar diretamente os recursos naturais como: qualidade da água, migração e deslocamento de espécies, ocasionando ainda fragmentação de habitats e erosão do solo (Uhl \& Vieira, 1989). Portanto, é indispensável a realização de estudos de planejamento e controle de estradas florestais, considerando o ponto de vista produtivo, os recursos naturais e a importância econômica para as grandes empresas do setor florestal.

\section{Aspectos metodológicos}

Considerando-se a abordagem da pesquisa, esta pode ser caracterizada como uma pesquisa mista, por abordar tanto aspectos qualitativos como quantitativos, enquadrando-se como um estudo de caso, em que se descreve e investiga uma situação real a partir de técnicas de investigação e teorias (Bogdan \& Biklen, 2013; Gil, 2009; Yin, 2005; Bruyne, Herman \& Schoutheete, 1977; Marconi \& Lakatos, 2017). A riqueza de informações possibilita maior conhecimento e entendimento quanto à problemática estudada.

O trabalho pode ser classificado como pesquisa aplicada, com propósito descritivo, em que, a partir do conhecimento.Buscou-se o desenvolvimento de soluções práticas, condizentes com a realidade estudada utilizando-se, também, de análise documental (Gerhardt \& Silveira, 2009; Gil, 2008; Yin, 2005; Moreira, 2005). 
Por isso, para um melhor entendimento do assunto, realizaram-se reuniões periódicas, com a equipe do setor. Dessa forma, foram levantados os dados qualitativos e discutidos os problemas enfrentados, e mapeadas empresas fornecedoras e parceiras para o desenvolvimento do sistema loT de rastreamento das atividades de terraplanagem que atendessem a necessidade de operação.

Foi realizado um estudo detalhado relacionado ao pagamento dos serviços e horas das máquinas de terraplanagem, com coleta, análise e interpretação de dados sem interferência do pesquisador. Para isso, foram analisados os formulários de "Partes Diárias", de modo a serem desenvolvidos dados quantitativos a respeito das máquinas de terraplanagem, como: tempo de trabalho, horímetro inicial e final e o número da máquina. Visou-se também compreender e analisar como o controle das horas de trabalho das máquinas era realizado pelos setores.

Compreendida a realidade, desenvolveu-se um sistema telemétrico para o controle das máquinas de terraplanagem, onde foram definidos quais os dados e indicadores chave deveriam ser desenvolvidos para monitorar a operação e como seriam armazenados em um software na nuvem. Por fim, foi feita uma comparação entre os dados preenchidos pelo operador na "Parte Diária" e os obtidos pelo sistema IoT com o objetivo de explicitar a diferença da hora-máquina de ambos os sistemas de controle e os benefícios com a aplicação da proposta.

\section{Resultados}

O presente estudo foi realizado em uma empresa produtora de celulose localizada no leste de Minas Gerais. O produto ofertado pela empresa é a celulose kraft branqueada de fibra curta de eucalipto. A empresa se divide em dois segmentos: o florestal e o industrial. No setor florestal realizamse as atividades de silvicultura, plantio e colheita da madeira; já o setor industrial é responsável pela produção da celulose.

O trabalho foi realizado no departamento de Infraestrutura Florestal, que tem como um dos seus objetivos a construção e manutenção das estradas florestais. Estas são usadas para o tráfego de mão de obra e dos meios de produção de implantação, proteção, colheita e transporte da madeira.

A empresa produtora de celulose contrata uma prestadora de serviço terceirizada para executar o serviço de terraplanagem. Atualmente, o sistema de controle dos serviços e horas trabalhadas pelas máquinas de terraplenagem é realizado através de um documento preenchido manualmente, denominado "Parte Diária".

Este documento é preenchido pelo operador do equipamento, que é um funcionário da empresa prestadora de serviço, que realiza o apontamento das horas parciais trabalhadas em frações de 15 minutos. Ou seja, a cada 15 minutos trabalhados, o operador deve marcar com um " $x$ " o campo correspondente. A Figura 1 representa um modelo de "Parte Diária". 
Figura 1

"Parte Diária" utilizada para o controle da hora-máquina

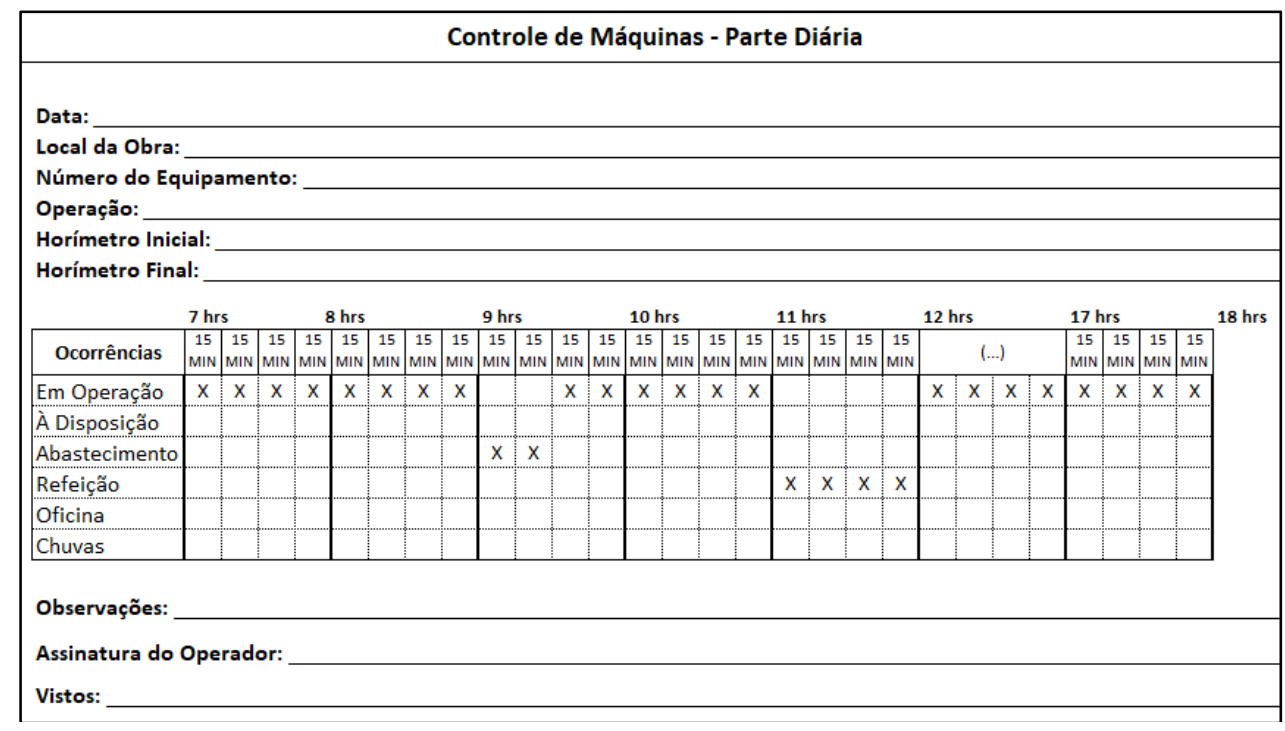

Fonte: As autoras (2020).

Após o preenchimento do documento, ao final do dia, o monitor de campo se desloca até o local onde se encontra a máquina e recolhe a "Parte Diária" do operador. Ele confere se todos os dados preenchidos estão corretos e, caso haja algum erro de preenchimento, esta "Parte Diária" é devolvida para o operador, que deve realizar as devidas correções.

Se todos os dados estiverem corretos, o monitor de campo assina o documento e o leva para o escritório onde o supervisor também deve conferir os dados e assiná-lo. Após a conferência do supervisor, o monitor de controle lança manualmente todas as Partes Diárias no sistema interno da empresa. Depois do lançamento dos dados, uma nova conferência é realizada pelos técnicos administrativos da empresa, para assim, ser realizado o pagamento. A Figura 2 a seguir apresenta o fluxograma do processo de pagamento da hora-máquina a partir da "Parte Diária". 
Figura 2

Fluxo do processo de pagamento da hora-máquina a partir da "Parte Diária"

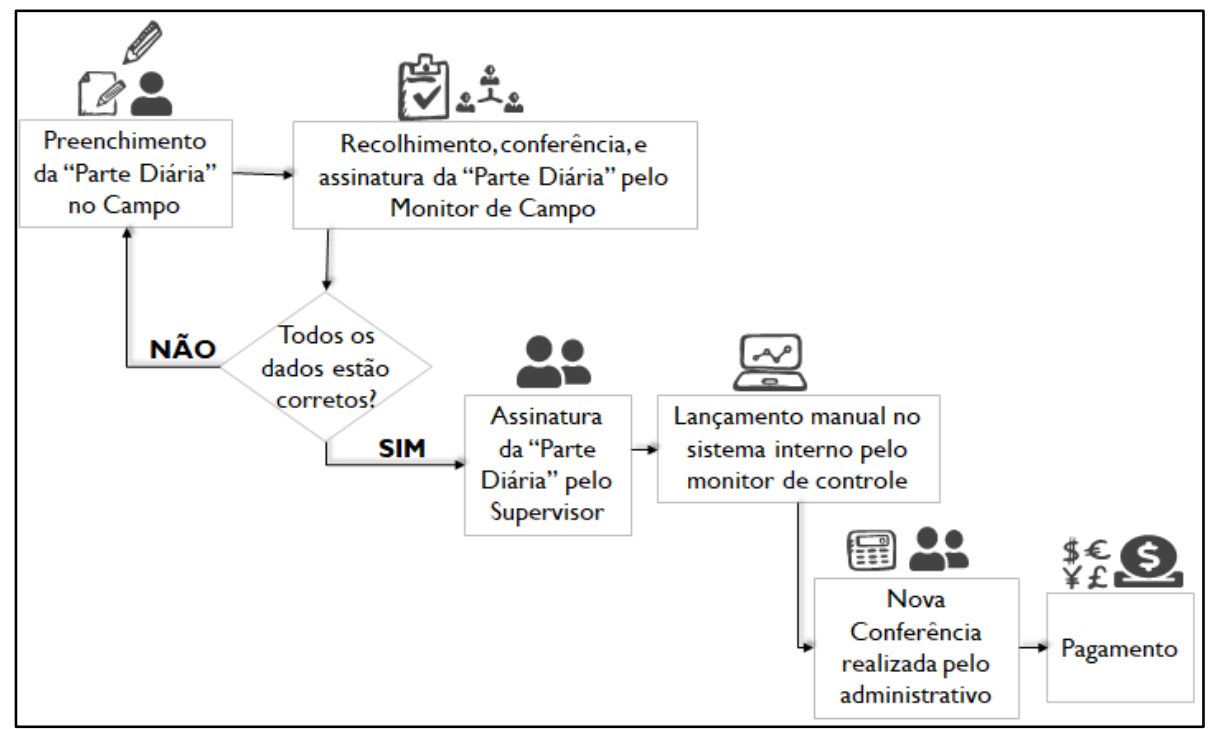

Fonte: As autoras (2020).

Tendo em vista que a marcação do horário de trabalho das máquinas é realizada manualmente pelo operador do equipamento, a empresa fica exposta, uma vez que as informações da jornada de trabalho dependerão do critério adotado pelo operador da empresa terceirizada na hora de preencher a "Parte Diária". Sendo assim, não é possível ter uma total segurança e controle das informações e do horário de trabalho das máquinas.

Outro ponto que também dificulta o processo é a localização das máquinas de terraplanagem. O monitor de campo deve se deslocar para buscar os formulários de "Partes Diárias", e como as máquinas se localizam em regiões dispersas, devido à demanda de trabalho da empresa, há uma dificuldade de logística para coletar os documentos preenchidos. Isso contribui para uma média elevada de quilômetros deslocados por mês. Atualmente, o departamento de infraestrutura florestal tem 5 monitores de campo, e cada monitor se desloca aproximadamente $3.200 \mathrm{~km} / \mathrm{mês}$.

Além disso, há o acúmulo destes documentos físicos na empresa, no qual existe uma série de pontos que ao serem analisados se torna evidente o quanto o documento em papel se tornou um gargalo dentro do processo, como:

- Manter um espaço físico para arquivamento;

- Risco do documento ser extraviado;

- Contratar um funcionário responsável pela organização;

- Gasto com papel e impressão;

- Perda de tempo no manuseio. 
Realizou-se uma pesquisa a fim de identificar a quantidade de formulários de "Partes Diárias" mantido, anualmente, dos equipamentos de infraestrutura florestal. Entre os anos de 2016 e 2019 foram mantidos o total de 13.435, 14.791, 17.048 e 19.332 documentos, respectivamente. O que indica alto volume de arquivos físicos a serem armazenados e mantidos, anualmente.

\subsection{Desenvolvimento do sistema loT de telemetria}

Inicialmente, utilizou-se a técnica do brainstorming (chuva de ideias) para levantar alternativas que permitissem o aumento de produtividade na operação e maior confiabilidade das informações. Diante do cenário mundial positivo para a implantação do sistema de rastreamento em veículos, e tendo em vista que outros departamentos da empresa já utilizam tal solução, optou-se por investir em um sistema de rastreamento para as máquinas de terraplanagem de infraestrutura florestal.

Inicialmente, definiram-se alguns critérios para a realização do orçamento e envio da proposta pela empresa fornecedora. Foi determinado que a transmissão do rastreamento utilizado fosse por telemetria satelital. Ou seja, seriam utilizadas diferentes constelações de satélites em órbita para coleta e transmissão dos dados, uma vez que a localização das máquinas é em área florestal onde há pouca cobertura de sinal de celular, sendo a transmissão via satélite o mais indicado por ter uma vasta abrangência e alta qualidade.

Estabelecida a alternativa a ser utilizada, iniciou-se a pesquisa de mercado para a busca de empresas especializadas neste tipo de sistema para a realização de orçamentos. Foi analisada a proposta de três empresas experientes em soluções para a gestão de frotas via telemetria veicular. Para a escolha da empresa a ser contratada, considerou-se um estudo de ganhos apresentados por elas, onde mostrava-se os benefícios e vantagens do sistema, e o custo de investimento.

Com isso, foi escolhida a empresa que apresentou o melhor estudo de ganhos, e por eventualidade o menor custo. Entre os benefícios contemplados no estudo de ganho, estão:

- Monitoramento em tempo real das máquinas;

- Controle operacional a distância;

- Monitoramento dos diversos sensores das máquinas tais como velocidade, RPM (rotações por minuto), horímetro e freio;

- Tempo exato de funcionamento das máquinas;

- Uma grande diversidade de relatórios que permite ao gestor identificar não conformidades e vícios de operação do veículo, permitindo feedback ao operador e plano de reforço de treinamento.

- Mapas e fotos por satelite;

- Armazenamentos dos dados por três anos nos servidores. 
A empresa contratada e a contratante, em conjunto, realizaram reuniões para definir as características do sistema de telemetria que seria desenvolvido, a fim de que fosse possível atender todas as máquinas. Tendo em vista que elas se localizam em diferentes regiões florestais com características distintas.

Definiu-se que, inicialmente, seria instalado o sistema de telemetria em apenas 27 máquinas de terraplanagem, para a fase de operação assistida. Esta fase consiste na presença de um técnico da empresa fornecedora do sistema de telemetria em todas as etapas do projeto. Isso é essencial para que haja uma correta implantação, uma vez que o técnico irá direcionar os operadores sobre as melhores práticas de utilização e operação do sistema, sendo capaz de identificar possíveis falhas na configuração do sistema, além de auxiliar na busca por soluções.

A indústria de celulose tem uma demanda sazonal de máquinas de terraplanagem, sendo que no período de março a agosto a demanda é maior devido ao período de seca. No período de chuva a demanda de máquinas é menor, pois as atividades são paralisadas de acordo com a intensidade da chuva e a quantidade de água existente no solo. Neste período, os operadores têm perda de boa parte da visibilidade, as máquinas perdem a aderência com o solo, atolam e correm risco de tombamento.

No período de desenvolvimento do trabalho, em julho, havia um total de 94 máquinas mobilizadas divididas em sete classes, em que classe refere-se ao tipo da máquina. A Tabela 1 apresenta o detalhamento das classes de máquinas mobilizadas no período e a quantidade total com a telemetria implantada.

Tabela 1

Quantidade de máquinas mobilizadas e com sistema de telemetria

$\begin{array}{ccc}\text { Classes de máquinas } & \begin{array}{c}\text { Quantidade de máquinas } \\ \text { mobilizadas }\end{array} & \begin{array}{c}\text { Quantidade de máquinas com } \\ \text { sistema de telemetria implantado }\end{array} \\ \text { Motoniveladora } & 25 & 11 \\ \text { Escavadeira Hidráulica } & 13 & 2 \\ \text { Pá Carregadeira } & 15 & 9 \\ \text { Retroescavadeira } & 10 & 5 \\ \text { Rolo Compactador } & 20 & 0 \\ \text { Trator de Esteira } & 5 & 0 \\ \text { Trator Pneu } & 6 & 27 \\ \text { Total } & 94 & 0\end{array}$

Fonte: As autoras (2020).

Conforme apresentado na Tabela 1, o sistema de telemetria foi instalado nas classes de equipamentos da motoniveladora, escavadeira hidráulica, pá carregadeira e retroescavadeira, não sendo instalado inicialmente na classe do rolo compactador, trator de esteira e pneu. 
O sistema de telemetria instalado nas 27 máquinas de terraplanagem é composto por um copiloto, um modem satelital, uma antena satelital, um tablet (interface homem-máquina) e um leitor RefAid. O sistema permite a leitura dos sensores da máquina transmitindo os dados automaticamente via satélite para um software na web. O software é acessado por usuários autorizados pelo departamento infraestrutura florestal e disponibiliza diversos tipos de relatórios detalhados e gerenciais sobre a utilização dos equipamentos.

Cada operador recebeu um crachá contendo o seu nome e sua matrícula. Ao iniciar uma operação, o operador da máquina deve identificar-se passando o seu crachá no leitor RefAid. Após se identificar, o operador deve apontar no tablet qual operação e atividade executarão e o nome do projeto que irá trabalhar.

Ao iniciar a operação, a tela do tablet exibe a atividade apontada e o tempo que o operador está executando-a. Há também um campo de mensagem, onde o operador pode relatar algum ocorrido durante o período de trabalho.

Ao final de cada dia, é gerado para cada equipamento que trabalhou um Workflow de aprovação. O Workflow de aprovação é uma página que é acessada dentro do software e contém todos os dados do equipamento ao finalizar o dia. Ao acessar a página do Workflow é necessário informar qual a máquina e qual dia que se deseja acessar as informações, sendo exibidas as seguintes informações do equipamento:

- Nome do operador que executou a operação;

- Projeto selecionado pelo operador;

- Rotação por minuto (RPM);

- Horímetro;

- Operação selecionada pelo operador;

- Data inicial e final da operação;

- Hora inicial e final da operação;

- Duração exata da operação.

Dentro desta página há também dois campos para aprovação, um do fornecedor do equipamento e outro do monitor de campo da empresa. O responsável pela aprovação deve marcar a opção OK se estiver de acordo com a informação apontada pelo operador e NOK caso contrário. O apontamento aprovado por ambos irá automaticamente para o sistema interno da empresa, que contabilizará o total de horas trabalhadas para realizar o pagamento da empresa prestadora de serviço. A Figura 3 apresenta o fluxograma do processo de pagamento das horas-máquinas a partir da telemetria. 
Figura 3

Fluxo do processo de pagamento a partir da telemetria

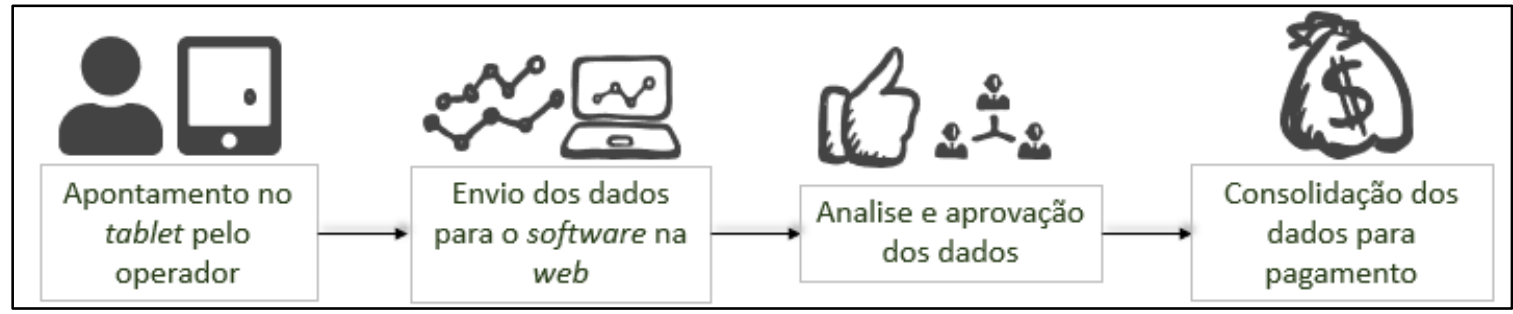

Fonte: As autoras (2020).

Conforme apresentado na Figura 3, o processo de pagamento da hora-máquina reduziu se comparado ao processo de pagamento da hora-máquina a partir da "Parte Diária" conforme mostrado na Figura 2.

\subsection{Impactos do sistema de loT de telemetria}

No decorrer do desenvolvimento do projeto alguns desafios e dificuldades foram encontrados. Para a consolidação do sistema, faz-se necessário que os dados transmitidos pela telemetria sejam condizentes com real trabalho das máquinas. Para a verificação dessas informações, estão sendo realizadas pela empresa inúmeras comparações entre formulários de "Partes Diárias" e os dados transmitidos pela telemetria. Quando é constatada uma diferença entre os dados, seja por falha do software ou por mal apontamento dos operadores, o técnico responsável pelo sistema é informado e, então, se realiza uma visita às máquinas a fim de corrigir tais imperfeições no software.

É imprescindível que os operadores das máquinas realizem de modo correto o apontamento das atividades que estão sendo realizadas, desde o início da operação até finalizá-la. O apontamento realizado de forma incorreta pelos trabalhadores é um dos obstáculos enfrentados, que requer treinamentos periódicos para aperfeiçoamento da operação.

Outro desafio encontrado é a cobertura de sinal para o envio das informações. Apesar de o sistema utilizar constelações de satélites de baixa órbita, destinado ao tráfego de dados para o envio das informações, verifica-se que quando o equipamento opera em uma área de sombra onde não há sinal, não é possível obter informações em tempo real do equipamento, uma vez que o rastreador não é capaz de transmitir as coordenadas. Contudo, o rastreador armazena os dados da máquina e assim que entra novamente em uma área de cobertura, envia automaticamente as informações ao servidor.

Para constatar os resultados obtidos com a implantação da telemetria, foi realizada uma comparação entre dados da "Parte Diária" com os dados da telemetria de três máquinas, sendo uma motoniveladora, uma pá carregadeira e uma retroescavadeira. Essas três máquinas foram escolhidas 
pois os dados transmitidos pela telemetria foram condizentes com o real trabalho dos operadores. Dessa forma, utilizou-se as duas formas de medições para as másquinas: telemetria e "Parte Diária".

Para a análise, utilizou-se sete dias variados, compreendidos entre o mês de julho/2019. A Tabela 2 apresenta a quantidade total de hora-máquina apontada na "Parte Diária", a quantidade total de hora-máquina transmitida pelo sistema telemétrico e a diferença desses dados em horas e percentual.

\section{Tabela 2}

Comparação entre os dados da telemetria e dos formulários de "Parte Diária"

\begin{tabular}{cccccc} 
Classe de Máquina & $\begin{array}{c}\text { Quantidade de Dias } \\
\text { Analisados }\end{array}$ & $\begin{array}{c}\text { Telemetria } \\
(\mathrm{h})\end{array}$ & $\begin{array}{c}\text { Parte Diária } \\
\text { (h) }\end{array}$ & $\begin{array}{c}\text { Diferença } \\
(\mathrm{h})\end{array}$ & \multicolumn{2}{c}{$\begin{array}{c}\text { Diferença } \\
(\%)\end{array}$} \\
Motoniveladora & 07 & 46,53 & 47,00 & 0,47 & $1,00 \%$ \\
Pá Carregadeira & 07 & 57,81 & 58,00 & 0,19 & $0,33 \%$ \\
Retroescavadeira & 07 & 54,56 & 55,00 & 0,44 & $0,81 \%$ \\
\hline
\end{tabular}

Fonte: As autoras (2020).

A partir dos dados da Tabela 2, realizou-se uma projeção da quantidade de horas que seria economizada por mês por cada uma dessas máquinas, considerando vinte e seis dias úteis mensais. Para realizar a simulação, primeiro encontrou-se o quanto seria economizado por dia, dividindo a diferença em horas encontrada pela quantidade de dias analisados (sete dias). Foi considerado apenas duas casas decimais para o arredondamento.

Após isso, multiplicou-se o valor encontrado por vinte e seis para obter a quantidade de horas que seria economizado por mês. Utilizando-se o valor médio pago da hora-equipamento, disponível na tabela custo horário do site Sobratema (data-base julho/2019), projetou-se o ganho mensal do projeto considerando uma motoniveladora, uma pá carregadeira e uma retroescavadeira em um mês. A Tabela 3 retrata estes dados.

\section{Tabela 3}

Projeção de ganho mensal do projeto considerando três máquinas

\begin{tabular}{ccccc} 
Classe de Máquina & $\begin{array}{c}\text { Economia/Dia } \\
(\mathrm{h})\end{array}$ & $\begin{array}{c}\text { Economia/Mês } \\
(\mathrm{h})\end{array}$ & $\begin{array}{c}\text { Valor Médio } \\
\text { Hora-Equipamento (R\$̦) }\end{array}$ & $\begin{array}{c}\text { Economia/Mês } \\
(\mathrm{R} \$ \text { ) }\end{array}$ \\
\hline Motoniveladora & 0,07 & 1,75 & $\mathrm{R} \$ 257,24$ & $\mathrm{R} \$ 450,17$ \\
Pá Carregadeira & 0,03 & 0,71 & $\mathrm{R} \$ 158,04$ & $\mathrm{R} \$ 112,21$ \\
Retroescavadeira & 0,06 & 1,63 & $\mathrm{R} \$ 140,17$ & $\mathrm{R} 228,48$ \\
\hline Total & & & & $\mathrm{R} \$ 790,86$
\end{tabular}

Fonte: As autoras (2020). 
Após a simulação da economia gerada por cada um desses equipamentos no mês, foi levantada a quantidade de motoniveladoras, pás carregadeiras e retroescavadeiras mobilizadas no mês de julho pela empresa. Considerou-se que todas essas máquinas trabalharam vinte e seis dias durante este período, assim projetou-se o ganho mensal dessas três classes como mostra a Tabela 4.

Tabela 4

Projeção de ganho mensal do projeto por classe de máquina

\begin{tabular}{cccc} 
Classe de máquina & $\begin{array}{c}\text { Quantidade de } \\
\text { Equipamentos }\end{array}$ & $\begin{array}{c}\text { Economia de um Equipamento } \\
(\mathrm{R} \$)\end{array}$ & $\begin{array}{c}\text { Economia } \\
\text { Total (R\$) }\end{array}$ \\
Motoniveladora & 25 & $\mathrm{R} \$ 450,17$ & $\mathrm{R} \$ 11.254,25$ \\
Pá Carregadeira & 15 & $\mathrm{R} \$ 112,21$ & $\mathrm{R} \$ 1.683,15$ \\
Retroescavadeira & 10 & $\mathrm{R} \$ 228,48$ & $\mathrm{R} \$ 2.284,80$ \\
\hline Total & & & $\mathrm{R} \$ 15.222,20$
\end{tabular}

Fonte: As autoras (2020).

Conforme mostra a Tabela 4, houve um ganho de produtividade de 0,8\% em um volume de $\mathrm{R} \$ 1.902 .322,33$ orçado para o uso de tais classes de máquinas no mês de julho. O que reafirma a importância do sistema, haja visto que reduções também são esperadas com o uso do sistema nas demais classes de máquinas - escavadeira hidráulica, rolo compactador, trator de esteira e trator pneu.

Adiciona-se, também, a projeção de economia para o ano e as demais melhorias alcançadas, tais como: monitoramento em tempo real das máquinas e de indicadores-chave, controle operacional a distância, levantamento de não conformidades, facilidade de armazenamento de dados e diminuição de armazenamento de documentos físicos e de custos de deslocamento para o recolhimento de formulário de "Parte Diária" preenchidos pelos operadores.

\section{Discussões}

A internet das coisas é considerada uma importante técnica de gestão de ativos (Ramos et al., 2008). Com eles, são gerados dados que, quando bem geridos, suportam decisões de qualidade às empresas (Hounsell et al., 2009). Os resultados desta pesquisa auxiliam nessa compreensão, pois sugerem os impactos da adoção do sistema de loT para rastreamento das máquinas.

Demonstrou-se que tal tipo de solução requer investimento para que a tecnologia seja desenvolvida. De modo que viabilize a infraestrutura de redes de dados, a governança adequada de dados, a padronização de uso de dispositivos móveis e o treinamento das equipes para a aplicação do novo procedimento e compreensão da nova cultura organizacional.

O sistema proporcionou, de um modo geral, melhor uso de recursos, sendo notados: simplificação de processos, além da diminuição de custos, de deslocamentos espaciais e de manutenção 
de documentos físicos. Por fim, tornou-se possível o monitoramento em tempo real de indicadores e os padrões de comportamento dos colaboradores quando em uso das máquinas. Dessa maneira, podemos fazer as seguintes proposições:

Proposição 1. o uso de loT demanda mudanças organizacionais para processamento e acesso a dados.

Proposição 2. o uso de loT requer governança de dados.

De acordo com Gubbi et al. (2013), a loT permite a medição, inferência e compreensão de indicadores através do uso de sensores nos ativos das empresas. Com isso, informações de qualidade são geradas e compartilhadas (Ding, Jin, Li \& Feng, 2020).

O controle da origem e da qualidade dos dados são essenciais para justificar o uso de loT. As intervenções manuais são comuns, o que requer o desenvolvimento de procedimentos sólidos de governança de dados (Brous, Janssen \& Herder, 2019; Ding, Jin, Li \& Feng, 2020). Diante disso, é possível estabelecer as seguintes proposições:

Proposição 3. 0 uso de loT requer que as pessoas desenvolvam novas habilidades.

Proposição 4. Ao adotar a loT, os processos organizacionais precisam de mudanças para coleta e uso de dados de diferentes fontes.

Os colaboradores precisam se adaptar às novas tecnologias, de modo que novas habilidades e maneiras de pensar sejam adquiridas. Com isso, surgem novas percepções sobre o processo de gestão (Brous, Janssen, Herder, 2019).

Os processos de gestão são executados por pessoas que trabalham em organizações com cultura social, sendo apoiadas em tecnologia para a tomada de decisões. O uso de uma nova tecnologia requer tanto o desenvolvimento de estruturas de dados complexas, quanto a mudança de práticas de gestão de ativos (Brous, Janssen \& Herder, 2019). A partir disso, pode-se definir que:

Proposição 5. 0 uso de loT requisita mudanças na maneira de coletar e usar dados de diferentes fontes.

Proposição 6. O uso de loT demanda a padronização da tecnologia.

A automatização dos processos de gestão ainda é um desafio, dada a complexidade dos dados processados em sistemas de loT, o que pode dificultar o compartilhamento de dados (Mihailovic, 2016). O uso loT atinge total êxito qundo podem ser coletados e diferentes fontes e eventos. 
Ao se introduzir uma nova tecnologia, é necessário repensar protocolos de transferência e segurança de dados, de modo que informações úteis sejam geradas. Para isso, as tecnologias necessitam ser padronizadas, de modo a garantir que sejam operacionalizadas (Ding, Jin, Li \& Feng, 2020). O uso de loT permite um melhor gerenciamento de ativos dada a forma de coleta e monitoramento de dados (Hua et al., 2014). Nesse sentido, é possível definir a última proposição:

\section{Proposição 7. A adoção de loT melhora o desempenho e a tomada de decisão.}

\section{Considerações finais}

Diante do exposto, pode-se observar que o presente estudo atingiu o seu objetivo inicial que consistiu em implantar sistema de loT para rastreamento em máquinas de terraplanagem a serviço de uma indústria de celulose. Com esta tecnologia, é possível medir todos os parâmetros dos equipamentos ocasionando uma maior segurança e redução de custo, pois, com os dados precisos, é possível entender se alguma etapa do processo está gerando desperdício e corrigi-lo.

Com a automatização das informações, os dados que antes eram lançados manualmente, um a um no sistema interno da empresa, serão lidos automaticamente pela plataforma, otimizando o tempo dos monitores e ocasionando em um aumento de produtividade. Outro ponto positivo é a eliminação do excesso de documentos em papéis, que corriam constantemente o risco de serem extraviados podendo gerar multas da fiscalização e auditória de trabalho. Além disso, a implantação deste sistema reduzirá no deslocamento dos monitores de campo, pois era necessário o deslocamento até as máquinas de terraplanagem para recolher os formulários de "Parte Diária".

É importante enfatizar, também, que o monitoramento em tempo real dos equipamentos serve de apoio constante à gestão. O que contribui de maneira vital para a competitividade e flexibilidade da organização e na busca por soluções e aprimoramentos necessários para um melhor desempenho.

O sistema telemétrico encontra-se implantado em apenas vinte e sete máquinas, sendo que a implantação nos restantes das máquinas só ocorrerá quando a transmissão dos dados for totalmente satisfatória. Sendo assim, a busca por soluções para sanar as dificuldades como, a falha na transmissão quando o equipamento está operando em área com sombra e sem sinal e o apontamento incorreto dos operadores, permanecerá até o aperfeiçoamento do sistema e da operação.

A comparação realizada entre, os dados transmitidos pela telemetria com os dados preenchidos nos formulários de "Parte Diária", nas três classes de máquinas: motoniveladora, pá carregadeira e retroescavadeira no período de sete dias, apresentou uma redução de hora-máquina de 1,0\%, 0,33\% e 0,81\% respectivamente, gerando uma redução de custo total de $\mathrm{R} \$ 15.222,20$ no uso de todos as máquinas das três classes, representando diminuição de $0,8 \%$ do orçamento do mês. 
Isso indica que o sistema pode ser usado em outras classes de equipamento, melhorando a projeção de redução de custos. Adicionamente, permitiu melhor monitoramento do de indicadores e máquinas, bem como o maior controle operacional à distância, além da facilidade no armazenamento de dados, diminuição de documentos físicos e de deslocamento para coleta de formulários preenchidos.

Quanto as contribuições teóricas, o estudo demonstrou que o uso de loT demanda mudanças organizacionais para governança, processamento e a acesso a dados, bem como requer o desenvolvimento de habilidades e formas de coleta e uso de dados. Adicionalmente, também são sugeridas adaptações na maneira de coletar e usar dados de diferentes fontes, bem como se faz necessário o uso padronizado de tecnologia. Todas as proposições indicam, portanto, que a loT melhora o desempenho e a tomada de decisão.

Como continuidade deste trabalho, recomenda-se realizar uma simulação do ganho anual que a empresa terá com a implantação do sistema de rastreio, e não apenas mensal, considerando todas as classes de equipamentos, uma vez que o estudo só considerou três de cinco classes, que tem a telemetria implantada. Assim, será possível obter o impacto anual do projeto para a indústria.

Dessa forma, conclui-se que se trata de um projeto de grande importância, levando-se em consideração as suas contrbuições teóricas e a melhoria na gestão das frentes de serviço e a redução de custo. Todos os benefícios somados fortificam a imagem da empresa, demonstrando a sua valorização com as novas tecnologias, e são facilmente replicáveis por empresas e indústrias do setor, melhorando o uso de recursos.

\section{Referências}

Ahlborn, T.M., Shuchman, R., Brooks, C.N., Harris, D.K., Burns, J.W., Roussi, C., Dobson, R., Vaghefi, K. \& Oats, R.C. (2010) An evaluation of commercially available remote sensors for assessing highway bridge condition. Journal of Bridge Engineering, v. 17, n. 6, p. 886-895.

Ardalan, A., Hammesfahr, J. and Pope, J. (1992) Total quality control: the repair facility. Industrial Management \& Data Systems, v. 92, n. 8, p. 7-10. DOI:

https://doi.org/10.1108/02635579210019802

Atzori, L. , A. lera , \& G. Morabito (2010). The Internet of Things: A Survey. Computer Networks, v. 54, n. 15, p. 2787-2805. DOI: https://doi.org/10.1016/j.comnet.2010.05.010

Backman, J. and Helaakoski, H. (2016), Evaluation of internet-of-things platforms for asset management. World Congress on Engineering Asset Management (WCEAM 2015), Springer International Publishing, p. 97-104.

Ben-Daya, M., Hassini, E., \& Bahroun, Z. (2020) Internet of things and supply chain management: a literature review. International Journal of Production Research, v. 57, n.15-16, p. 4719-4742. DOI: https://doi.org/10.1080/00207543.2017.1402140 
Bogdan, R. C., \& Biklen, S. K. (2013) Investigação qualitativa em educação: uma introdução à teoria e aos métodos. 12. ed. Porto: Porto Editora.

Boos, D., Guenter, H., Grote, G. \& Kinder, K. (2013) Controllable accountabilities: the internet of things and its challenges for organisations. Behaviour \& Information Technology, v. 32, n. 5, p. 449467. DOI: https://doi.org/10.1080/0144929X.2012.674157

Bramucci, M. (2001) Determinação e qualificação de fatores de influencia sobre a produtividade de "Harvesters" na colheita de madeira. 50 f. Dissertação (Mestrado em Recursos Florestais) Escola Superior de Agricultura Luiz de Queiroz, Piracicaba.

Brous, P., Janssen, M. \& Vilminko-Heikkinen, R. (2016), Coordinating decision-making in data management activities: a systematic review of data governance principles. International Conference on Electronic Government and the Information Systems Perspective, Springer, p. $115-125$

Brous, P., Janssen, M., Schraven, D., Spiegeler, J. \& Duzgun, B.C. (2017), Factors influencing adoption of iot for data-driven decision making in asset management organizations. International Conference on Internet of Things, Big Data and Security, p. 70-79. DOI: https://doi.org/10.5220/0006296300700079

Brous, P., Janssen, M. \& Herder, P. (2019) Internet of Things adoption for reconfiguring decisionmaking processes in asset management. Business Process Management Journal, v. 25, n. 3, p. 495-511.

Bruyne, P.; Herman, J.; \& Schoutheete, M. (1977) Dinâmica da pesquisa em ciências sociais: os pólos da prática metodológica. Rio de Janeiro: F. Alves, 251 p. DOI: https://doi.org/10.1108/BPMJ11-2017-0328

Campo, R. F. d. (2014) Avaliação da densidade de estradas em propriedades rurais no sul do ES. Monografia (Engenharia Florestal)- Universidade Federal do Espírito Santo, Espírito Santo.

Chase, R. B., Jacobs, R., \& Aquilano, N. J.(2006) Administração da produção para vantagem competitiva. 10 ed. Porto Alegre: Bookman.

Comunello, A. C. (2014) Planejamento e controle da produção: um estudo em uma indústria do Oeste do Paraná. Trabalho de Conclusão de Curso (Engenharia de Produção) - Universidade Tecnológica Federal do Paraná, Medianeira.

Decker, C. , Berchtold, M., Chaves, L. W. F., Beigl, M., Roehr, D., Riedel, T. , M., Beuster, Herzog, T., \& Herzig, D. (2008). Cost-Benefit Model for Smart Items in the Supply Chain. The Internet of Things Lecture Notes in Computer Science, v. 4952, p. 155-172. DOI: https://doi.org/10.1007/978-3-540-78731-0_10

Ding, Y., Jin, M., Li, S. \& Feng, D. (2020) Smart logistics based on the internet of things technology: an overview, International Journal of Logistics Research and Applications. DOI: https://doi.org/10.1080/13675567.2020.1757053

Eisenhardt, K.M. \& Zbaracki, M.J. (1992) Strategic decision making. Strategic Management Journal, v. 13, n.S2, p. 17-37. 
Eisenhardt, K.M. (1989) Building theories from case study research. The Academy of Management Review, v. 14, n. 4, p. 532-550. DOI: https://doi.org/10.2307/258557

Ellis, S., Morris, H. D., \& Santagate, J. (2015) loT-Enabled Analytic Applications Revolutionize Supply Chain Planning \& Execution. International Data Corporation (IDC).

Florence, D. and Queree, C. (1993) Traceability - Problem or Opportunity?. Logistics Information Management, v. 6, n. 4, p. 3-8. DOI: https://doi.org/10.1108/09576059310045899

Furley, T. H., Mello, F. A. D., \& Siqueira, J. B. L. (2018) Principais questões ambientais causadas pelos efluentes de fábricas de celulose da América Latina. O Papel. São Paulo, v. 79, n. 4, p 70-77.

Gehardt, T. E., \& Silveira, D. T. (2009) Métodos de pesquisa. Apostila UAB/UFRGS, Porto Alegre: Editora da UFRGS

Gil, A. C. (2008) Métodos e técnicas de pesquisa social. 6. ed. São Paulo: Editora Atlas.

GIL, A. C. (2009) Como elaborar projetos de pesquisa. 4. ed. São Paulo: Atlas.

Greeves, B. (1994) Scada uses radio to bridge the gap. Sensor Review, v. 14, n. 2, p. 31-34. DOI: https://doi.org/10.1108/EUM0000000004252

Gubbi, J., Buyya, R., Marusic, S. \& Palaniswami, M. (2013). Internet of things (IoT): a vision, architectural elements, and future directions. Future Generation Computer Systems, v. 29, n. 7, p. 1645-1660. DOI: https://doi.org/10.1016/j.future.2013.01.010

Hounsell, N.B., Shrestha, B.P., Piao, J. \& McDonald, M. (2009) Review of urban traffic management and the impacts of new vehicle technologies. IET Intelligent Transport Systems, v. 3, n. 4, p. 419428. DOI: https://doi.org/10.1049/iet-its.2009.0046

Hua, L., Junguo, Z. \& Fantao, L. (2014) Internet of things technology and its applications in smart grid. Indonesian Journal of Electrical Engineering and Computer Science, v. 12, n. 2, p. 940-946. DOI: https://doi.org/10.11591/telkomnika.v12i2.4178

Ibá (2017). Relatório 2017.

Ibá (2018). Sumário executivo 2018.

Koo, D., Piratla, K. \& Matthews, C.J. (2015), Towards sustainable water supply: schematic development of big data collection using internet of things (IOT). Procedia Engineering, v. 118, p. 489-497. DOI: https://doi.org/10.1016/j.proeng.2015.08.465

Kotler, P. (2011) Administração de marketing: análise, planejamento e controle. V. 1, 2 e 3. São Paulo: Atlas.

Lemos, A., \& Josgrilberg, F. (2009) Comunicação e mobilidade: Aspectos Socioculturais das Tecnologias Móveis de Comunicação no Brasil. Salvador: EDUFBA.

Li, Z., Guo, H., Barenji, A. V., Wang, W. M., Guan, Y., \& Huang, G. Q. (2020) A sustainable production capability evaluation mechanism based on blockchain, LSTM, analytic hierarchy process for supply chain network. International Journal of Production Research. DOI:

https://doi.org/10.1080/00207543.2020.1740342 
Lopes, E. S. (2012) Colheita de baixo volume individual. Rev. Opiniões, Ribeirão Preto, v. 27, p. 21.

Machado, C. C., Silva, E. N., \& Pereira, R. S. (2008) O setor florestal brasileiro. In: MACHADO, C. C. (Coord.) Colheita florestal. 2. ed. Viçosa: Editora UFV, cap. 1, p. 15-42.

Malinovski, J. R. et al. (2004) Código de prática para estradas de uso florestais. Otacílio Costa: Malha Viária Logística de Estradas.

Marconi, M. D. A., \& Lakatos, E. M.(2017) Metodologia científica. 7. ed. São Paulo: Atlas.

Mihailovic, A. (2016), "Liberalising deployment of internet of things devices and services in large scale environments", Wireless Personal Communications, Vol. 92 No. 1, pp. 33-49.

Moreira, S. V. (2005) Análise documental como método e como técnica. In: DUARTE, Jorge; BARROS, Antonio (Org.). Métodos e técnicas de pesquisa em comunicação, São Paulo: Atlas, p. 269-279.

Navarro, R. M. S., Navarro, F. M. S., \& Tambourgi, E. B. (2007) Estudo de diferentes processos de obtenção da pasta celulósica para fabricação de papel. Revista ciências \& tecnologias, São Paulo, ano $1, \mathrm{n} 1$.

Neto, G. C. O., Costa, I., Sousa, W. C., Amorim, M. P. C. \& Godinho Filho, M. (2019) Adoption of a telemetry system by a logistics service provider for road transport of express cargo: a case study in Brazil, International Journal of Logistics Research and Applications, v. 22, n. 6, p. 592613. DOI: https://doi.org/10.1080/13675567.2018.1564253

Núñez-Merino, M., Maqueira-Marín, J. M., Moyano-Fuentes, J., \& Martínez-Jurado, P. J. (2020). Information and digital technologies of Industry 4.0 and Lean supply chain management: a systematic literature review. , v.58, n.16, p. 5034-5061. DOI: https://doi.org/10.1080/00207543.2020.1743896

Oliveira, R. J., Machado, C.C., Souza, A.P., \& Leite, H.G. (2006) Avaliação técnica e econômica da extração de madeira de eucalipto com "clambunck skidder". Revista Árvore, Viçosa, v. 30, n.2, p. 267-275. DOI: https://doi.org/10.1590/S0100-67622006000200014

Osório, E. G. (2007) Indústria de papel e celulose: estudo de caso da implantação da VCP florestal no extremo sul do Rio Grande do Sul. Monografia (Especialização-Curso de Economia, Ciências Econômica)- Universidade Federal de Santa Catarina, Florianópolis.

Parida, A., Kumar, U., Galar, D. and Stenström, C. (2015), Performance measurement and management for maintenance: a literature review. Journal of Quality in Maintenance Engineering, v. 21, n. 1, p. 2-33. DOI: https://doi.org/10.1108/JQME-10-2013-0067

Penna, E. S., Machado, C. C., Souza, A. P., Silva, E., \& Silva, E. N. (2011) Avaliação ergonômica de modelos de cabos aéreos utilizados na extração florestal. Revista Árvore, Viçosa, v. 35, n. 3, p. 565-571. DOI: https://doi.org/10.1590/2179-8087.064013

Pinagé, E. R., \& Matricardi, E. A. T. (2015) Detecção da infraestrutura para exploração florestal em Rondônia utilizando dados de Sensoriamento Remoto. Floresta e ambiente, v. 22, n. 3, p. $377-$ 390. 
Ramos, C., Augusto, J.C. and Shapiro, D. (2008) Ambient intelligence-the next step for artificial intelligence. IEEE Intelligent Systems, v. 23, n. 2, p. 15-18. DOI: https://doi.org/10.1109/MIS.2008.19

Rathore, M.M., Ahmad, A., Paul, A. \& Thikshaja, U.K. (2016), Exploiting real-time big data to empower smart transportation using big graphs. 2016 IEEE Region 10 Symposium (TENSYMP), p. 135139. https://doi.org/10.1109/TENCONSpring.2016.7519392

Rocha, M. B. (2016) Fatores e Riscos ergonômicos na colheita florestal mecanizada. Dissertação (Mestrado em Ciências Florestais)- Universidade Estadual do Sudoeste da Bahia, Vitória da Conquista.

Ross, D. F. 2016. Introduction to Supply Chain Management Technologies. Boca Raton, FL: St Lucie Press.

Santos, P. H. A., Souza, A. P., Marzano, F. L. C., \& Minette, L .J. (2013) Produtividade e custos de extração de madeira de eucalipto com clambunck skidder. Revista Árvore, Viçosa, v. 37, n.3, p. 511-518. DOI: https://doi.org/10.1590/S0100-67622013000300014

Schettino, S., Minette, L. J., \& Souza, A. P. (2015) Correlação entre volumetria de florestas de eucalipto e produtividade e custos de máquinas de colheita de madeira. Rev. Árvore, Viçosa, v. 39, n.5, Viçosa, p.935-942. DOI: https://doi.org/10.1590/0100-67622015000500016

SFB. (2012) Brasil com Florestas: oportunidades para o desenvolvimento de uma economia florestal e a reestruturação necessária do setor, Brasília.

Silveira, P. R., \& Santos, W. E. (2002) Automação e controle discreto. 4. ed. São Paulo: Érica.

Teixeira, F. C. R., Oliveira, M. C. D., \& Helleno, A. L. (2014) Telemetria Automotiva via Internet Móvel. Revista Ciência e Tecnologia, [S.I.], v. 16, n. 28/29, fev. 2014.

Uhl C., \& Vieira I. C. G. (1989) Ecological impacts of selective logging in the Brazilian Amazon: a case study from the Paragominas Region of the State of Pará. Biotropica, p. 98-106. DOI: https://doi.org/10.2307/2388700

Verdouw, C. N. , Wolfert, J., Beulens, A. J. M. , \& Rialland, A. (2016) Virtualization of Food Supply Chains with the Internet of Things. Journal of Food Engineering, v. 176, p. 128-136. DOI:https://doi.org/10.1016/j.jfoodeng.2015.11.009

Xu, L. D. , W. He , \& S. Li . (2014). Internet of Things in Industries: A Survey. IEEE Transactions on Industrial Informatics, v. 10, n. 4, p. 2233-2243. DOI: https://doi.org/10.1109/TII.2014.2300753

Yin, R. K. (2005) Estudo de caso: planejamento e métodos. 3. ed. Porto Alegre: Bookman. 212 p. 